\title{
Review Article \\ The Use of Dried Blood Spots for the Quantification of Antihypertensive Drugs
}

\author{
Alexander Chernonosov \\ Institute of Chemical Biology and Fundamental Medicine, Siberian Branch of Russian Academy of Sciences, Academician Lavrentiev \\ Avenue 8, Novosibirsk 630090, Russia \\ Correspondence should be addressed to Alexander Chernonosov; alexander.chernonosov@niboch.nsc.ru
}

Received 30 March 2018; Revised 9 June 2018; Accepted 2 July 2018; Published 1 August 2018

Academic Editor: Jan Åke Jönsson

Copyright (C) 2018 Alexander Chernonosov. This is an open access article distributed under the Creative Commons Attribution License, which permits unrestricted use, distribution, and reproduction in any medium, provided the original work is properly cited.

\begin{abstract}
Hypertension or high blood pressure is a harbinger of cardiovascular diseases. There are several classes of drugs used to treat hypertension. This review discusses the use of dried blood spots (DBSs) for the quantification by mass spectrometry (MS), tandem mass spectrometry (MS/MS), or, in some cases, by fluorescence detection methods the following antihypertensive medications: angiotensin-converting enzyme inhibitors (ramipril, ramiprilat, captopril, and lisinopril); angiotensin II receptor antagonists (valsartan, irbesartan, losartan, and losartan carboxylic acid); calcium channel blockers (verapamil, amlodipine, nifedipine, pregabalin, and diltiazem); $\alpha$ blockers (guanfacine, doxazosin, and prazosin); $\beta$ blockers (propranolol, bisoprolol, atenolol, and metoprolol); endothelin receptor antagonists (bosentan and ambrisentan); and statins (simvastatin, atorvastatin, and rosuvastatin).
\end{abstract}

\section{Introduction}

Cardiovascular disease is the number one cause of death in the world [1]. Hypertension is the main attributable risk factor of death and is responsible for approximately half of the cases of cerebrovascular and ischemic heart diseases $[2,3]$. Moreover, blood pressure over $140 / 90 \mathrm{mmHg}$ is a cause of heart failure and chronic kidney disease in $20 \%$ and $23 \%$ of cases, respectively [3-5]. As a rule, high blood pressure is widespread among the elderly [3]. Blood pressure above $140 / 90 \mathrm{mmHg}$ is observed in more than $50 \%$ of people aged 60 years and $75 \%$ over the age of 70 years $[6,7]$, and the treatment of hypertension is becoming an increasingly urgent problem with the ageing of society [3]. On the other hand, blood pressure $>140 / 90$, despite treatment with a diuretic and two other antihypertensive drugs of various classes, is defined as drug-resistant hypertension $[8,9]$. In the USA, patients who need to take more than four antihypertensive drugs to achieve a normal blood pressure level are also considered resistant hypertensives [10].

Nevertheless, most people with hypertension require more than one drug for effective control of the disease.
Because hypertension can develop in different biochemical ways, various classes of antihypertensive drugs have been developed. Diuretics, calcium channel blockers (CCBs), $\alpha$ blockers, $\beta$ blockers (BBs), and inhibitors of the reninangiotensin system are used for initial antihypertension therapy [11]. In addition, angiotensin-converting enzyme (ACE) inhibitors, angiotensin II receptor antagonists (ARAs), and endothelin receptor antagonists (ERAs) have been used for antihypertensive therapy over the past 20 years $[12,13]$. CCBs, ARAs, and BBs have been the most prescribed antihypertensive medications [14-16]. Treatment of most patients (67.92 $\%)$ includes more than one drug. The most commonly used combination of medications has been $\mathrm{CCB}+\mathrm{BB}+\alpha$ blocker (7.55 \%) [13]. For effective treatment, patients should follow the indications for taking a given drug and the prescribed dose [17]. The big problem is that $~ 50 \%$ of all patients with heart disease do not adhere to their prescribed regimen [1820]. Often, such patients are referred to as patients with apparently resistant hypertension [9]. Patients, who ignore or do not adhere to their prescribed medication [21], more often as a result show considerable morbidity, higher costs of care, and mortality [22-26]. Many clinicians fail to assess blood pressure regularly or effectively titrate and regulate the dose 
of drugs; these shortcomings result in ineffective treatment [27].

The use of dried blood spots (DBSs) can simplify the methods for determining the concentrations of drugs. The DBS sampling technique is minimally invasive, and capillary blood can be obtained from a finger prick with a lancet by the patients themselves or guardians with minimal training. Such a sampling technique is also suitable even for small children [28] and is ideal for routine clinical testing [29] or helps with recruitment of subjects for preclinical or clinical studies [30]. Besides, DBSs reduce to a minimum the risk of infection with HIV and other infectious pathogens [31]. Moreover, DBSs offer a simpler storage and easier transfer by mail to the assigned laboratory, preventing unnecessary costs [32-34]. They should be well desiccated after sampling (2-3 hours minimum). The combined advantage of the above benefits coupled with improved analytical instrumental capability [35] has been recognized for the use of this methodology for various applications including therapeutic drug monitoring [36-38], toxicokinetic studies [39], and preclinical or clinical pharmacokinetic studies [30, 39-43].

In the current paper, quantification of major classes of antihypertensive drugs and statins in DBS by liquid chromatography with mass spectrometry (LC-MS), liquid chromatography with tandem mass spectrometry (LC-MS/MS), and fluorescence detection methods in recent years are reviewed. Parameters of analysis that can influence sensitivity were analyzed: types of used DBS cards, mass-spectrometers and detection modes, and the diameter of a disk punched for analysis, linear concentration range, elution solvents, extraction procedures, recovery, and stability of DBS samples.

\section{DBS Analysis}

The technique of DBS was first suggested by Dr. Robert Guthrie in the 1960s for diagnosis of phenylketonuria by neonatal screening [44]. In this sampling technique, a small volume of blood $(30-50 \mu \mathrm{L})$ is collected on filter paper or DBS cards based on a cellulose matrix with specific pore size and thickness $[45,46]$.

Several types of DBS cards are usually used for blood sampling in quantitative drug analysis. The FTA DMPK-A/ FTA card and FTA DMPK-B/ FTA elute card are coated and contain additives for analysis of nucleic acids [47]. Four types of DBS cards are uncoated: FTA DMPK-C/ 31ETF, Whatman 903, and two cards manufactured by Ahlstrom (226 and 237) [45]. After drying, a DBS sample is stored under conditions suitable for the analyte, usually at room temperature (RT) or $4^{\circ} \mathrm{C}$. The sample could be sent by mail to a remote analytical laboratory if required.

A disk of a certain diameter is then punched and subjected to an extraction procedure. This procedure is simple: a suitable solvent is applied to dissolve the analyte in the punched disk of a DBS. Sometimes a second step, involving solid phase extraction (SPE) or liquid extraction (LLE), is required for further analysis [45]. The analysis involves a small amount of an analyte (as a consequence of the small volume of blood in a DBS): nanogram or even picogram amounts are suitable for fluorescence, MS, or MS/MS detection methods. Thus, the combination of the DBS technique with such methods allows for analyzing low concentrations of drugs in blood samples [30].

MS/MS includes selected-reaction monitoring (SRM) and multiple-reaction monitoring (MRM) modes, when only the transition between a selected precursor and product ions is detected. This approach enables excluding other possible ions and thus significantly improves sensitivity and selectivity of the assay [74].

At present, triple quadrupole (QQQ) mass-spectrometers remain the gold standard for quantification [74, 75], but quadrupole time-of-flight (QTOF) mass-spectrometers are also employed in many DBS studies.

Some analytical parameters are important for comparing assays of the same medication. First of all, there is the type of DBS card, size of the disk punched for analysis, and recovery of the analyte from a DBS; these directly affect the lower limit of quantitation (LLOQ). Assay sensitivity also depends on equipment type and detection mode. The extraction procedure with a suitable elution solvent is often characterized by simplicity. The linear concentration range and stability of DBS samples describe the assay in general. Table 1 summarizes the methods of quantification of antihypertensive drugs in DBS, published from 2009 to 2017. The search for the articles was performed in the PubMed database by means of the following keywords: DBS or "dried blood spots" with the name of medications.

DBS sampling was reported for several classes of antihypertensive drugs, such as ACE inhibitors, ARAs, CCBs, alpha blockers, BBs, and ERAs as well as for statins. Although statins are not antihypertensive drugs themselves, they are often used as part of a combination therapy and enhance the antihypertensive effect of ACE inhibitors or CCBs, but not $\mathrm{BBs}$ or diuretics [76]. In most of the reviewed studies, statins have been simultaneously analyzed along with antihypertensive drugs.

\section{ACE Inhibitors}

These drugs block the peripheral conversion of angiotensin I to angiotensin II [77]. The DBS technique has predominantly been used by Tanna and coauthors for quantification of ramipril [48, 50, 51], captopril [53], and lisinopril [50]. Ramipril is the most studied medication among ACE inhibitors and is stable up to 84 days at RT with good recovery ( 90\%) from Whatman 903 and Ahlstrom 226 cards. Ramipril has been detected by QTOF mass-spectrometer with $417.2384 \mathrm{~m} / \mathrm{z}$. The only difference in the elution procedure was the extraction solvent: $300 \mu \mathrm{L}$ of methanol $(\mathrm{MeOH})$ [50] or $150 \mu \mathrm{L}$ of $\mathrm{MeOH} / \mathrm{H}_{2} \mathrm{O}(70: 30, \mathrm{v} / \mathrm{v})[48,49,51]$, and the latter showed better recovery. Lisinopril was analyzed in the same assay [50] in TOF mode at $406.2336 \mathrm{~m} / \mathrm{z}$. Captopril has been quantitated with similar results in SRM and TOF modes [53]. For this analysis, the Whatman 903 card was pretreated by dithiothreitol (DDT) and extraction solvent containing 10\% v/v $200 \mathrm{mM}$ DTT. It was necessary to reconstitute captopril from its disulfide dimer in blood 


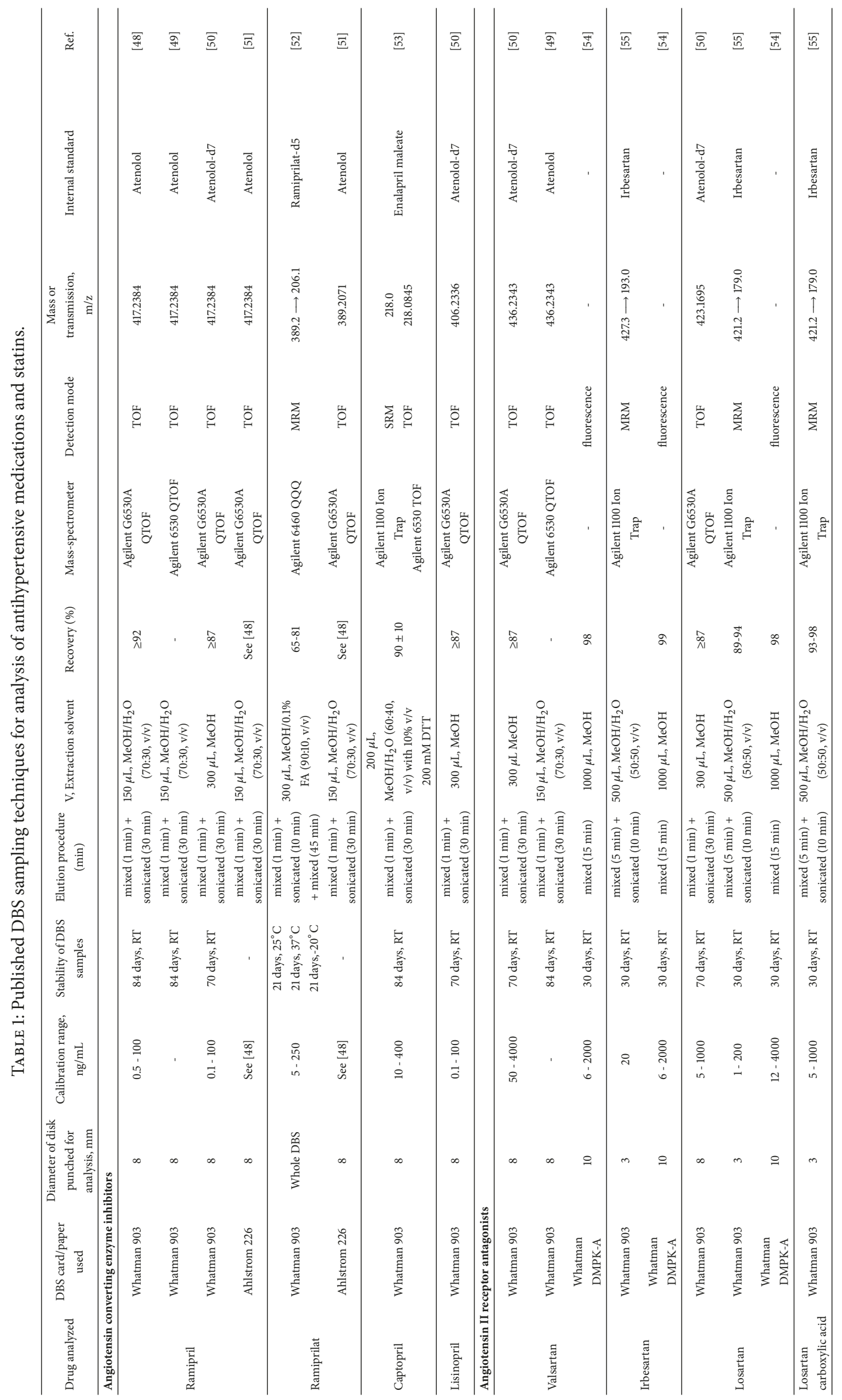




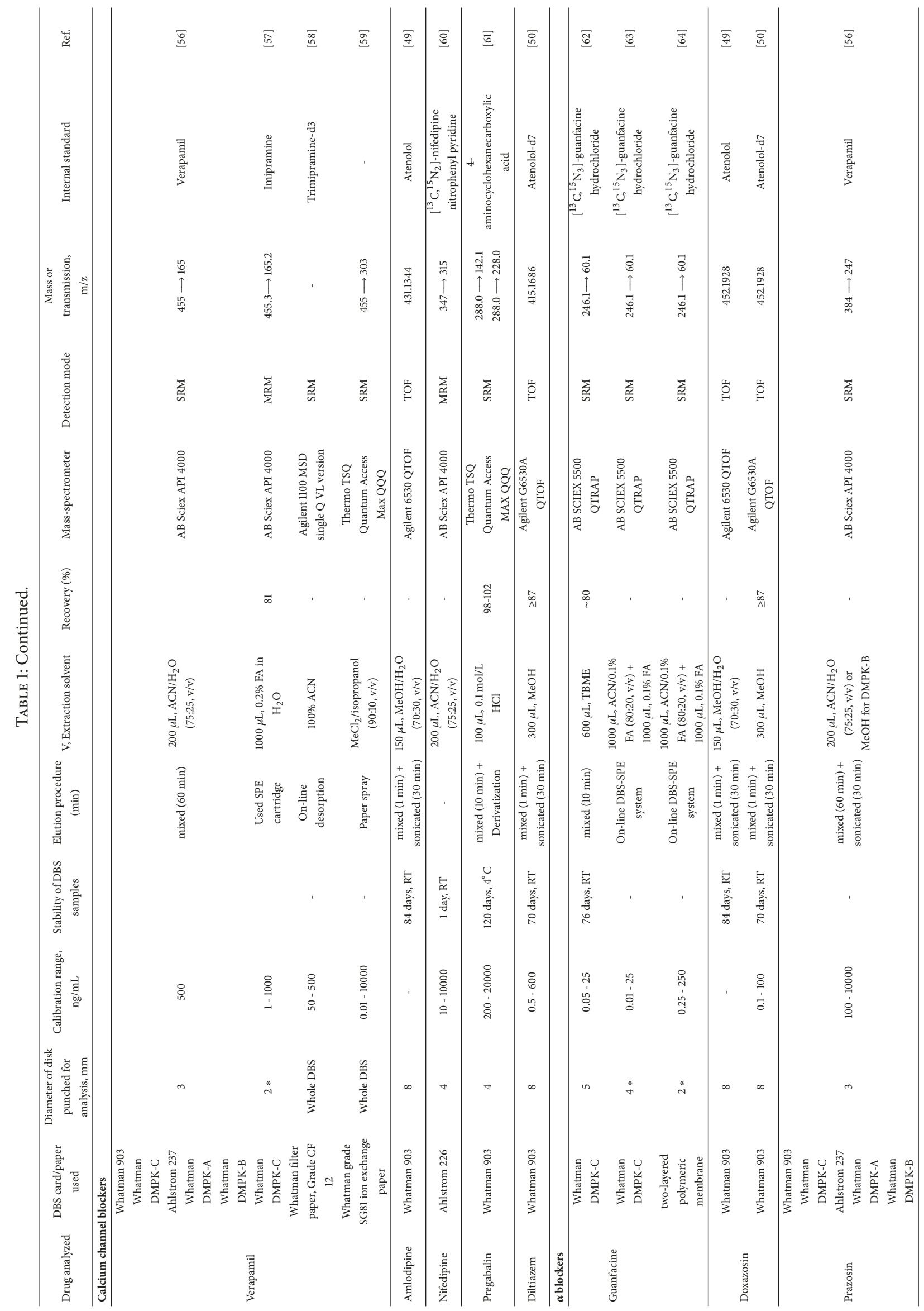




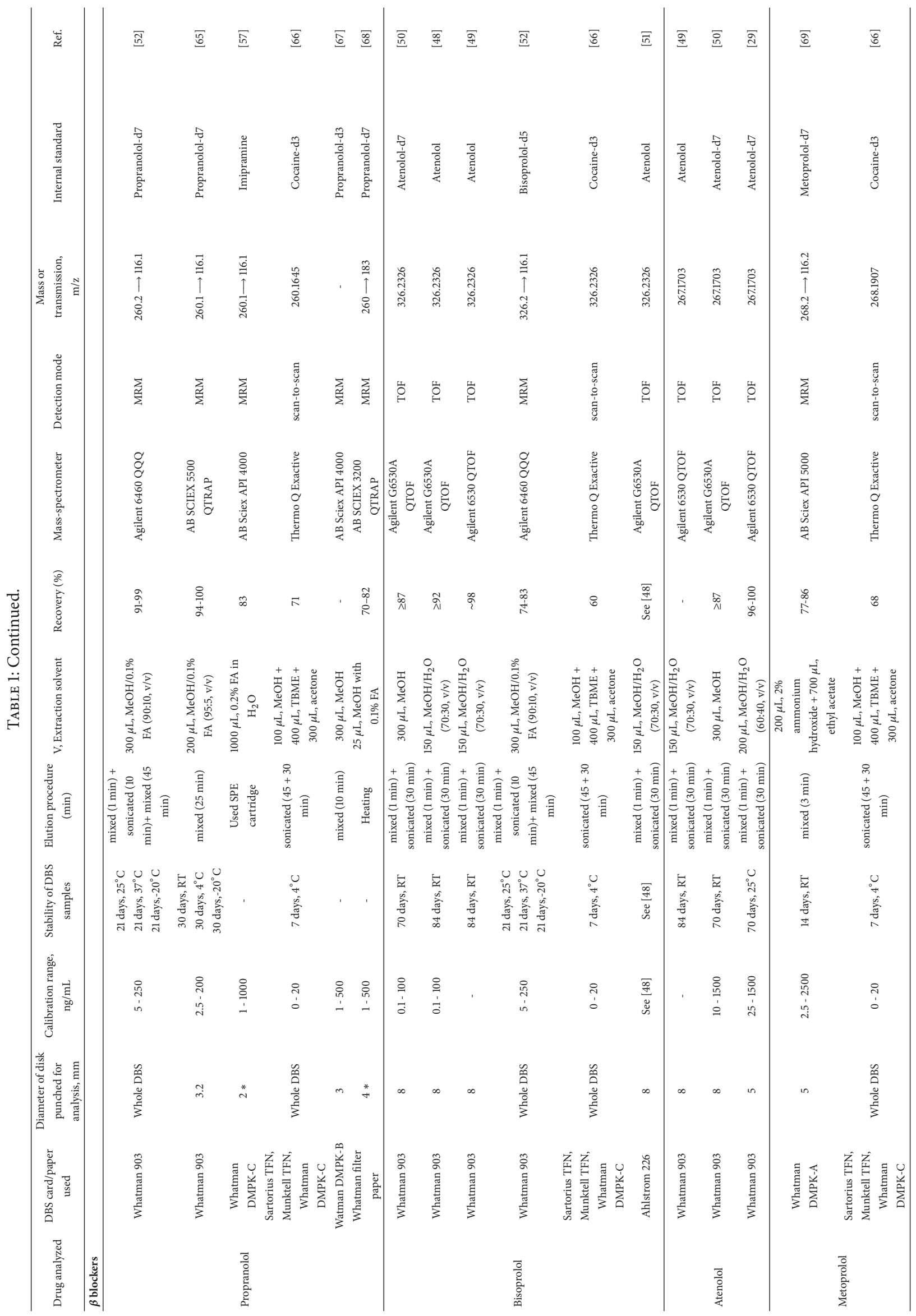




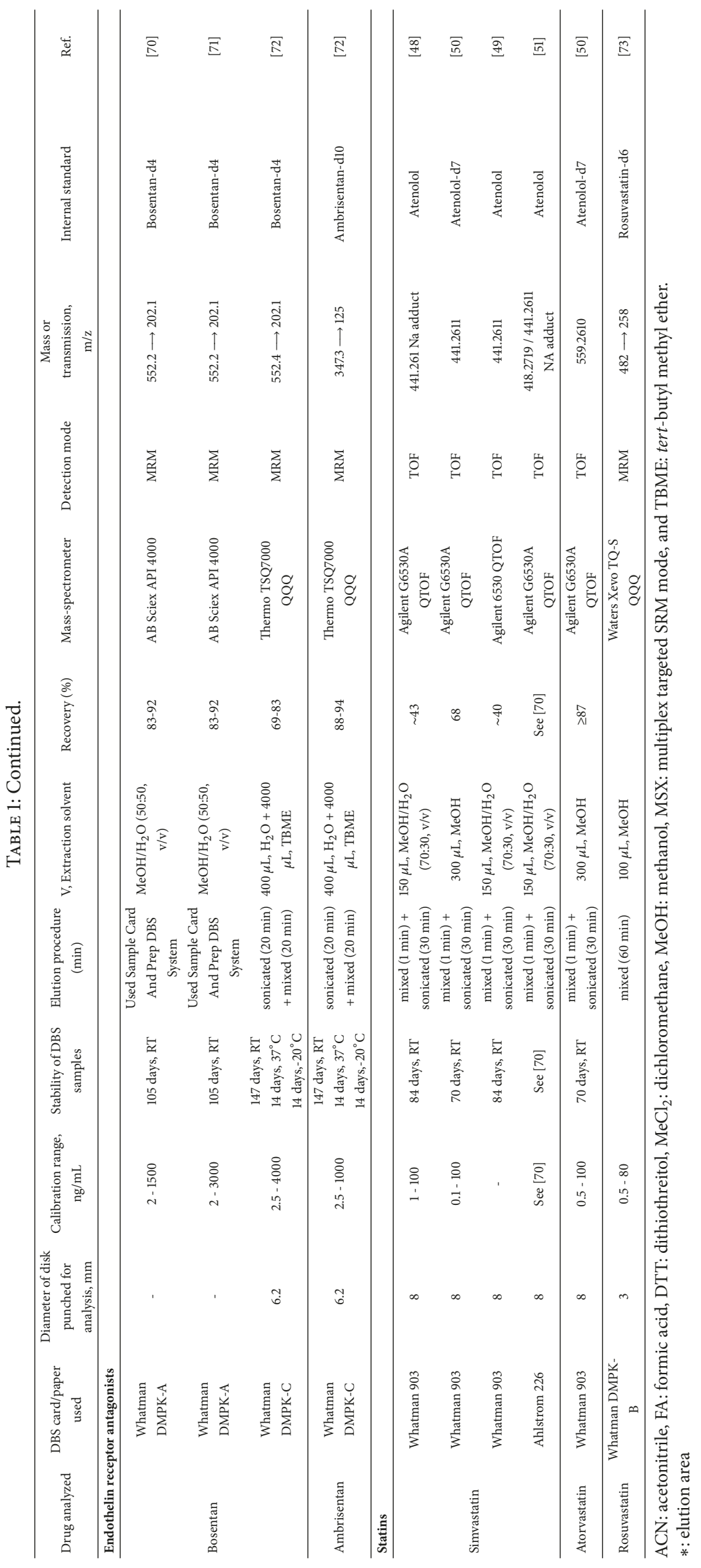


samples. Ramiprilat is an active metabolite of ramipril and can be analyzed alone [52] or together with ramipril [51]. These studies cannot be completely compared because the assay was not described in detail in [51] and simply cited a paper where method of ramipril analysis was reported [48].

\section{ARAs}

ARAs specifically and selectively block type I angiotensin, a receptor of the renin-angiotensin system, by displacing angiotensin II from it [78, 79]. The presence of fluorescent functional groups in the molecular structure of ARA has allowed researchers to develop fluorescent assays for simultaneous quantification of valsartan, irbesartan, and losartan in DBS [54]. In this case, $1000 \mu \mathrm{L}$ of $\mathrm{MeOH}$ served as an extraction solvent with 98-99 \% recovery. The assay sensitivity for valsartan was better than that reported in studies on MS detection in TOF mode $[49,50]$. Irbesartan has been detected in MRM mode only as an internal standard (IS), but losartan detection in the MRM mode enables investigators to achieve LOQ of $1 \mathrm{ng} / \mathrm{mL}$ [55]. Losartan carboxylic acid is a more potent active metabolite of losartan [80], which has also been detected in MRM mode [55] with excellent recovery (93-98\%). DBS samples of valsartan, irbesartan, losartan, and losartan carboxylic acid are stable up to 84, 30, 70, and 30 days at $\mathrm{RT}$, respectively.

\section{CCBs}

Antihypertensive properties of CCBs are mediated by their ability to disrupt the movement of calcium ions through membrane channels. Several classes of CCBs can be distinguished based on their chemical structure: 1.4dihydropyridines (amlodipine, pregabalin, and nifedipine), phenylalkylamine (verapamil), benzothiazepines (diltiazem), and others.

Verapamil has been used as a model analyte to develop and improve methods of analysis in DBS. For example, the following are direct assays via on-line desorption of DBS [58]: flow-through desorption of DBS without an LC column [57], the paper spray ambient ionization method for direct analysis of biological samples [59], and desorption electrospray ionization (DESI) that operates at atmospheric pressure [56].

In these works, verapamil has been detected by MS/MS with transitions $455 \longrightarrow 303$ or $455 \longrightarrow 165 \mathrm{~m} / \mathrm{z}$. The paper spray method involving Whatman grade SG81 ion exchange paper shows the best sensitivity: down to $0.01 \mathrm{ng} / \mathrm{mL}$. In this case, in contrast to other studies, the spot was cut out as a triangle $(10 \times 5 \mathrm{~mm})$ [59]. Different solvents have been applied for elution: acetonitrile $(\mathrm{ACN}) / \mathrm{H}_{2} \mathrm{O}, 100 \% \mathrm{ACN}$, and dichloromethane/isopropanol $(90: 10, \mathrm{v} / \mathrm{v})$, but only for the flow-through desorption method was the recovery $(81 \%)$ reported [57]. The stability of DBS samples in these studies has not been evaluated.

Amlodipine [49] and diltiazem [50] have been simultaneously analyzed along with ramipril and other medications under the conditions described above for ramipril.
To improve LOQ, the derivatization protocol with npropyl chloroformate [81] has been developed for pregabalin quantitation in DBS [61]. The simple elution procedure with $0.1 \mathrm{M} \mathrm{HCl}$ yielded approximately $100 \%$ recovery. Nevertheless, LOQ was still high: $200 \mathrm{ng} / \mathrm{mL}$.

The DBS technique has been applied to a study on the ways to increase stability of photosensitive compounds [60]. Nifedipine was used as a model analyte for this purpose. Extraction of nifedipine was performed on the Ahlstrom 226 card using micronic 96-well plates and silicone mats with a polytetrafluoroethylene film [60] by means of $200 \mu \mathrm{L}$ of $\mathrm{ACN} / \mathrm{H}_{2} \mathrm{O}(70: 30, \mathrm{v} / \mathrm{v})$. It was shown that photosensitive compounds are more stable in DBSs than in blood or plasma under exposure to light.

\section{Alpha Blockers}

These are a class of antihypertensive drugs that has a vasodilating effect as a result of predominantly blocking $\alpha$ adrenergic receptors. Guanfacine, like verapamil, has been chosen as a model analyte in several studies on improvement of DBS assays [62-64]. The analyte has been detected on the same equipment in SRM mode with transition $246.1 \longrightarrow$ $60.1 \mathrm{~m} / \mathrm{z}$. At first, the standard DBS technique was developed with good stability of DBS samples (76 days at RT), a simple extraction procedure (10 min mixing) involving $600 \mu \mathrm{L}$ of tert-butyl methyl ether (TBME), with $\sim 80 \%$ recovery from a Whatman DMPK-C card and the concentration range from 0.05 to $25 \mathrm{ng} / \mathrm{mL}$ [62]. Then, methods of on-line desorption from a Whatman DMPK-C card [63] and from a two-layered polymeric membrane [64] were developed. The best sensitivity was achieved with the on-line DBS-SPE system based on Whatman DMPK-C cards. Doxazosin was simultaneously analyzed along with ramipril and other medications under the conditions described above for ramipril $[49,50]$. Prazosin was analyzed by the DESI technique [56]. Several types of filter card were tested in that work: Whatman 903, FTA DMPK-A, FTA DMPK-B, and FTA DMPK-C. Nonetheless, the LOQ shown by the DESI technique was $100 \mathrm{ng} / \mathrm{mL}$ : not as low as in a comparable electrospray ionization assay [56].

\section{BBs}

This is a large group of drugs that block the binding sites on $\beta$-adrenergic receptors. Antihypertensive medications of this class are most often subjected to the DBS technique, for example, propranolol and bisoprolol. These two have been tested on different types of filter paper, including Sartorius TFN and Munktell TFN [66]. The DBS samples were stable on a Whatman 903 card for up to 30 and 84 days for propranolol [65] and bisoprolol [48, 49], respectively. The extraction procedure for bisoprolol was a standard one-mixing and sonication-whereas propranolol was extracted via on-line desorption [57] and paper heating [68]. The best recovery rates, up to $98-99 \%$, for propranolol and bisoprolol were achieved with an extraction solvent consisting of $\mathrm{MeOH}$ or $\mathrm{MeOH} / \mathrm{H}_{2} \mathrm{O}$ mixtures in different combination with or without $0.1 \%$ formic acid (FA). In contrast, extraction with $100 \mu \mathrm{L}$ 
of $\mathrm{MeOH}, 400 \mu \mathrm{L}$ of TBME, and $300 \mu \mathrm{L}$ of acetone resulted in $71 \%$ and $60 \%$ recovery for propranolol and bisoprolol, respectively [66]. The difference lies in the main detection mode: MRM for propranolol and TOF for bisoprolol. In two studies, atenolol was simultaneously assayed along with ramipril and other medications under conditions described above for ramipril $[49,50]$. In the third paper, the DBS technique was generally the same and yielded similar results [29]. Metoprolol was extracted in two ways: by means of 100 $\mu \mathrm{L}$ of $\mathrm{MeOH}, 400 \mu \mathrm{L}$ of TBME, and $300 \mu \mathrm{L}$ of acetone or with $200 \mu \mathrm{L}$ of $2 \%$ ammonium hydroxide and $700 \mu \mathrm{L}$ of ethyl acetate. The last solvent with simple 3 min mixing showed better recovery, up to $86 \%$. In this case, the concentration range was $2.5-2500 \mathrm{ng} / \mathrm{mL}$, with 14 -day stability of DBS samples at RT.

\section{ERAs}

These drugs block endothelin receptors; ambrisentan acts on endothelin A receptors, whereas bosentan affects both endothelin A and B receptors. Only these two medications have been analyzed by the DBS sampling technique, and detection has been carried out in MRM mode for both. Elution of bosentan was performed using a Sample Card and Prep DBS System by means of $\mathrm{MeOH} / \mathrm{H}_{2} \mathrm{O}(50: 50, \mathrm{v} / \mathrm{v})$ $[70,71]$ or simultaneously with ambrisentan in a standard assay with $400 \mu \mathrm{L} \mathrm{H}_{2} \mathrm{O}$ and $4 \mathrm{~mL}$ of TBME [72]. The recovery in these two approaches was approximately the same, but bosentan recovery in the case of $\mathrm{MeOH} / \mathrm{H}_{2} \mathrm{O}(50: 50$, v/v) was a little better. DBSs of both analytes were found to be stable for up to 147 days at RT.

\section{Statins}

These agents inhibit 3-hydroxy-3-methyl-glutaryl-coenzyme A reductase; as a result, the blood levels of total cholesterol, low-density lipoproteins, and triglycerides decrease, while the concentration of antiatherogenic high-density lipoproteins increases. The combination of statins with antihypertensive drugs increases the activity of the latter. Therefore, statins are often quantified simultaneously with antihypertensive drugs. Just as the analytes above, simvastatin [48-51] and atorvastatin [50] have been analyzed simultaneously with other medications under conditions described in a previous section for ramipril. The only problem with simvastatin is recovery, which did not exceed $68 \%$. Rosuvastatin was assayed by 2 -dimensional analytical-scale chromatography with at-column dilution enabling the injection of large sample volumes of organic extracts of DBS [73]. Analysis of rosuvastatin was performed in MRM mode with simple mixing extraction by means of $100 \mu \mathrm{L}$ of $\mathrm{MeOH}$ in the concentration range $5-80 \mathrm{ng} / \mathrm{mL}$.

\section{Effects of Blood Properties}

Aside from the parameters of analysis described above, a researcher should take into account the effects of blood properties, such as hematocrit, blood volume, and blood distribution in a DBS as well as sample quality, which may have a direct impact on quantitative analysis. Hematocrit is the most important parameter influencing the accuracy and precision of DBS analysis [82, 83]. Hematocrit varies with gender, health status, and age and slightly with ethnicity [74]. Moreover, capillary blood tends to have higher hematocrit (e.g., 61\%) than venous blood does [30]. Reference ranges slightly vary among sources but are typically $40-50 \%$ for adult men and $35-45 \%$ for adult women $[30,84,85]$. In a hospital population, $95 \%$ of routine hematocrit measurements yield values between $23 \%$ and $48 \%$ [74]. Hematocrit affects the spot size and formation, homogeneity of the spot, drying time, recovery of the analyte, and reproducibility and robustness of the assay $[46,74]$. Therefore, it is important to evaluate the hematocrit effect during validation of an assay [46]. In several research papers, investigators have considered the influence of hematocrit on quantitative determination of analyte concentrations. No significant impact of hematocrit on the quantification was found in the hematocrit range 35-65\% for bosentan [70], 38-45\% for bosentan and ambrisentan [72], $23-43 \%$ for propranolol [65], 41-48\% for guanfacine [62], and $20-50 \%$ for losartan and losartan carboxylic acid [55] and the hematocrit range of $35-55 \%$ for ramipril, lisinopril, valsartan, losartan, diltiazem, doxazosin, bisoprolol, atenolol, simvastatin, and atorvastatin [50]. The impact of hematocrit was evaluated in a follow-up to [64], where unacceptable bias was detected for guanfacine at hematocrit values of $30 \%$ and $60 \%$, but acceptable accuracy and precision results were obtained at hematocrit $45 \%$ [86]. Besides, alternative approaches have been used for correction of hematocrit values: a mathematical equation for pregabalin [61], a number of consecutive analyses of the same blood spot for verapamil and propranolol [57], and analysis of the entire blood spot sample for ramiprilat, propranolol, and bisoprolol [52]. In some cases, the hematocrit effect was not studied because the authors assumed that hematocrit variation is likely to be within the "normal" hematocrit range in adults [29, 48]. In other studies, the hematocrit effect has not been considered.

Because it has been observed that the DBS area decreases nearly linearly when hematocrit increases, the spot size and homogeneity of spots also influence quantification results $[74,84]$. Usually, the spotted blood volume is between 15 and $40 \mu \mathrm{L}$. Accordingly, the impact of the blood spotting volume on DBS analysis has been investigated in several works: blood volume of 20, 25, and $30 \mu \mathrm{L}$ [70]; 20, 30, and $40 \mu \mathrm{L}[29,48,50,53] ; 10$ to $40 \mu \mathrm{L}[69] ; 15,20$, and $25 \mu \mathrm{L}$ [62]; and 10,15 , and $20 \mu \mathrm{L}$ [55]. In all cases, accuracy and precision were less than $15 \%$ within the tested range, and therefore accurate pipetting during preparation of DBS may not be necessary. To minimize the influence of spot homogeneity on measurement, the same location of a spot (center) $[29,48-$ $51,53,54,61-63,69,73]$ or the whole spot $[52,66]$ has been punched. No difference between punching the central area and punching an offcenter site of DBS has been observed for pregabalin [61] and guanfacine [62].

In many cases, analyte concentration can differ between capillary and venous blood [87] as well as between plasma and DBS. Thus, a comparison is recommended as part of the 
assay validation [30]. Such a study has been conducted for guanfacine, showing on average $20 \%$ higher concentrations in DBS than in plasma, but an $11.6 \%$ to $23.2 \%$ decrease in concentration was observed when whole blood rather than plasma was spiked with guanfacine [62]. The analyte concentrations in plasma show a significant correlation with DBS concentrations with a conversion factor (slope) of 1.73 for propranolol [65], 1.58 for ambrisentan, and 1.52 for bosentan [72]. Bosentan DBS and plasma concentrations have also been compared in a Bland-Altman plot, and a limited systematic difference between the measurements was found [71]. For rosuvastatin, quantification methods for either plasma or DBS have been developed, but comparison experiments for plasma and DBS samples have not been carried out [73]. For nifedipine, only photostability has been compared between DBS and liquid aqueous and biological matrices [60].

Samples during validation assays are collected in the laboratory. If samples are collected in the laboratory from volunteers [29, 48-51] or rats [52,54], they are usually spotted correctly. For home sample collection, the proportion of unsatisfactory samples is $19 \%$ [49]. This figure can reach more than $30 \%[74,88]$ and may pose a problem for clinical application because the assays are validated by a correct technique for spotting of samples.

\section{Conclusions}

Interest in the DBS technique as an easy blood sampling method for monitoring of antihypertensive drug concentrations is increasing continuously. The main limitation of the DBS technique is sensitivity, which is expected to improve with the growing availability of MS and MS/MS equipment in clinical and scientific laboratories for analysis of antihypertensive drugs. Among the reviewed works, medications have in general been analyzed in MRM mode on QQQ mass-spectrometers or in TOF mode on QTOF mass-spectrometers. The elution procedure in many cases consists of mixing and sonication in an extraction solvent with suitable recovery. Most popular types of filter paper are Whatman 903 or Whatman DMPK-C/-A cards. Almost all antihypertensive drugs show great stability in DBS samples for weeks or even months. An automated DBS technique and on-line desorption of DBS have been devised in some studies to enable analysis of a large number of samples. The major variables of the DBS technique are hematocrit and differences in drug concentration measured in blood, blood plasma, and DBS. Validation experiments are necessary for new DBS assays, but not all the published drug assays involving DBS include full validation; this situation can be considered the main obstacle for their broad application.

\section{Conflicts of Interest}

The author declares that there are no conflicts of interest regarding the publication of this paper.

\section{Acknowledgments}

This work was supported by a Program of RAS, "Basic Research for Biomedical Technology" 2018-2020.

\section{References}

[1] World Health Organization, "Cardiovascular Diseases (CVDs)," 2017, http://www.who.int/mediacentre/factsheets/fs317/en/.

[2] C. M. Lawes, S. V. Hoorn, and A. Rodgers, "Global burden of blood-pressure-related disease, 2001," The Lancet, vol. 371, no. 9623, pp. 1513-1518, 2008.

[3] D. J. Campbell, M. Mcgrady, D. L. Prior et al., "Most individuals with treated blood pressures above target receive only one or two antihypertensive drug classes," Internal Medicine Journal, vol. 43, no. 2, pp. 137-143, 2013.

[4] S. M. Dunlay, S. A. Weston, S. J. Jacobsen, and V. L. Roger, "Risk factors for heart failure: a population-based case-control study," American Journal of Medicine, vol. 122, no. 11, pp. 1023-1028, 2009.

[5] M. K. Haroun, B. G. Jaar, S. C. Hoffman, G. W. Comstock, M. J. Klag, and J. Coresh, "Risk factors for chronic kidney disease: a prospective study of 23,534 men and women in Washington County, Maryland," Journal of the American Society of Nephrology, vol. 14, no. 11, pp. 2934-2941, 2003.

[6] R. S. Vasan, A. Beiser, S. Seshadri et al., "Residual lifetime risk for developing hypertension in middle-aged women and men," Journal of the American Medical Association, vol. 287, no. 8, pp. 1003-1010, 2002.

[7] N. D. Wong, V. A. Lopez, G. L'Italien, R. Chen, S. E. J. Kline, and S. S. Franklin, "Inadequate control of hypertension in US adults with cardiovascular disease comorbidities in 2003-2004," JAMA Internal Medicine, vol. 167, no. 22, pp. 2431-2436, 2007.

[8] G. Mancia, R. Fagard, K. Narkiewicz et al., "2013 ESH/ESC Guidelines for the management of arterial hypertension: The Task Force for the management of arterial hypertension of the European Society of Hypertension (ESH) and of the European Society of Cardiology (ESC)," Journal of hypertension, vol. 31, no. 7, pp. 1281-1357, 2013.

[9] E. Berra, M. Azizi, A. Capron et al., "Evaluation of adherence should become an integral part of assessment of patients with apparently treatment-resistant hypertension," Hypertension, vol. 68, no. 2, pp. 297-306, 2016.

[10] A. V. Chobanian, G. L. Bakris, and H. R. Black, "Seventh report of the Joint National Committee on prevention, detection, evaluation, and treatment of high blood pressure," Hypertension, vol. 42, no. 6, pp. 1206-1252, 2003.

[11] G. Rimoy, M. Justin-Temu, and C. Nilay, "Prescribing Patterns and cost of antihypertensive drugs in private hospitals in Dar es Salaam, Tanzania," East and Central African Journal of Pharmaceutical Sciences, vol. 11, no. 3, 2009.

[12] M. C. Cáceres, P. Moyano, H. Fariñas et al., "Trends in antihypertensive drug use in spanish primary health care (19902012)," Advances in Pharmacoepidemiology \& Drug Safety, vol. 04, no. 01, 2015.

[13] N. Jarari, N. Rao, J. R. Peela et al., "A review on prescribing patterns of antihypertensive drugs," Clinical Hypertension, vol. 22, article 7, 2015

[14] H. Xu, Y. He, L. Xu, X. Yan, and H. Dai, “Trends and patterns of five antihypertensive drug classes between 2007 and 2012 in China using hospital prescription data," International Journal of 
Clinical Pharmacology and Therapeutics, vol. 53, no. 6, pp. 430437, 2015.

[15] P.-H. Liu and J.-D. Wang, "Antihypertensive medication prescription patterns and time trends for newly-diagnosed uncomplicated hypertension patients in Taiwan," BMC Health Services Research, vol. 8, article no. 133, 2008.

[16] M. Beg, S. Dutta, A. Varma et al., "Study on drug prescribing pattern in hypertensive patients in a tertiary care teaching hospital at Dehradun, Uttarakhand," International Journal of Medical Science and Public Health, vol. 3, no. 8, pp. 922-926, 2014.

[17] S. Tanna and G. Lawson, Analytical Chemistry for Assessing Medication Adherence, Elsevier, Amsterdam, Netherlands, 2016.

[18] A. Shroufi and J. W. Powles, "Adherence and chemoprevention in major cardiovascular disease: A simulation study of the benefits of additional use of statins," Journal of Epidemiology and Community Health, vol. 64, no. 2, pp. 109-113, 2010.

[19] S. Baroletti and H. Dell'Orfano, "Medication adherence in cardiovascular disease," Circulation, vol. 121, no. 12, pp. 14551458, 2010.

[20] I. M. Kronish and S. Ye, "Adherence to cardiovascular medications: Lessons learned and future directions," Progress in Cardiovascular Diseases, vol. 55, no. 6, pp. 590-600, 2013.

[21] P. M. Ho, J. A. Spertus, F. A. Masoudi et al., "Impact of medication therapy discontinuation on mortality after myocardial infarction," JAMA Internal Medicine, vol. 166, no. 17, pp. 18421847, 2006.

[22] P. M. Ho, C. L. Bryson, and J. S. Rumsfeld, "Medication adherence: its importance in cardiovascular outcomes," Circulation, vol. 119, no. 23, pp. 3028-3035, 2009.

[23] J. B. Garner, "Problems of nonadherence in cardiology and proposals to improve outcomes," American Journal of Cardiology, vol. 105, no. 10, pp. 1495-1501, 2010.

[24] H. B. Bosworth, B. B. Granger, P. Mendys et al., "Medication adherence: A call for action," American Heart Journal, vol. 162, no. 3, pp. 412-424, 2011.

[25] A. La Caze, G. Gujral, and W. N. Cottrell, "How do we better translate adherence research into improvements in patient care?" International Journal of Clinical Pharmacy, vol. 36, no. 1, pp. 10-14, 2014.

[26] I. Barat, F. Andreasen, and E. M. S. Damsgaard, "Drug therapy in the elderly: What doctors believe and patients actually do," British Journal of Clinical Pharmacology, vol. 51, no. 6, pp. 615622, 2001.

[27] T. A. Kotchen, "The search for strategies to control hypertension," Circulation, vol. 122, no. 12, pp. 1141-1143, 2010.

[28] H. C. Pandya, N. Spooner, and H. Mulla, "Dried blood spots, pharmacokinetic studies and better medicines for children," Bioanalysis, vol. 3, no. 7, pp. 779-786, 2011.

[29] G. Lawson, E. Cocks, and S. Tanna, "Quantitative determination of atenolol in dried blood spot samples by LC-HRMS: A potential method for assessing medication adherence," Journal of Chromatography B, vol. 897, pp. 72-79, 2012.

[30] W. Li and F. L. S. Tse, "Dried blood spot sampling in combination with LC-MS/MS for quantitative analysis of small molecules," Biomedical Chromatography, vol. 24, no. 1, pp. 4965, 2010.

[31] S. P. Parker and W. D. Cubitt, "The use of the dried blood spot sample in epidemiological studies," Journal of Clinical Pathology, vol. 52, no. 9, pp. 633-639, 1999.
[32] R. G. Boy, J. Henseler, R. Mattern, and G. Skopp, "Determination of morphine and 6-acetylmorphine in blood with use of dried blood spots," Therapeutic Drug Monitoring, vol. 30, no. 6, pp. 733-739, 2008.

[33] G. la Marca, S. Malvagia, L. Filippi, F. Luceri, G. Moneti, and R. Guerrini, "A new rapid micromethod for the assay of phenobarbital from dried blood spots by LC-tandem mass spectrometry." Epilepsia, vol. 50, no. 12, pp. 2658-2662, 2009.

[34] A. Chernonosov, "Quantification of warfarin in dried rat plasma spots by high-performance liquid chromatography with tandem mass spectrometry," Journal of Pharmaceutics, vol. 2016, Article ID 6053295, 6 pages, 2016.

[35] S. Tanna and G. Lawson, "Analytical methods used in conjunction with dried blood spots," Analytical Methods, vol. 3, no. 8, pp. 1709-1718, 2011.

[36] P. M. Edelbroek, J. V. D. Heijden, and L. M. L. Stolk, "Dried blood spot methods in therapeutic drug monitoring: Methods, assays, and pitfalls," Therapeutic Drug Monitoring, vol. 31, no. 3, pp. 327-336, 2009.

[37] S. AbuRuz, J. Millership, and J. McElnay, "Dried blood spot liquid chromatography assay for therapeutic drug monitoring of metformin," Journal of Chromatography B, vol. 832, no. 2, pp. 202-207, 2006.

[38] J. van der Heijden, Y. de Beer, K. Hoogtanders et al., "Therapeutic drug monitoring of everolimus using the dried blood spot method in combination with liquid chromatographymass spectrometry," Journal of Pharmaceutical and Biomedical Analysis, vol. 50, no. 4, pp. 664-670, 2009.

[39] M. Barfield, N. Spooner, R. Lad, S. Parry, and S. Fowles, "Application of dried blood spots combined with HPLC-MS/MS for the quantification of acetaminophen in toxicokinetic studies," Journal of Chromatography B, vol. 870, no. 1, pp. 32-37, 2008.

[40] P. Wong, R. Pham, B. A. Bruenner, and C. A. James, "Increasing efficiency for dried blood spot analysis: Prospects for automation and simplified sample analysis," Bioanalysis, vol. 2, no. 11, pp. 1787-1789, 2010.

[41] N. Spooner, R. Lad, and M. Barfield, "Dried blood spots as a sample collection technique for the determination of pharmacokinetics in clinical studies: considerations for the validation of a quantitative bioanalytical method," Analytical Chemistry, vol. 81, no. 4, pp. 1557-1563, 2009.

[42] P. Patel, S. Tanna, H. Mulla, V. Kairamkonda, H. Pandya, and G. Lawson, "Dexamethasone quantification in dried blood spot samples using LC-MS: The potential for application to neonatal pharmacokinetic studies," Journal of Chromatography B, vol. 878, no. 31, pp. 3277-3282, 2010.

[43] M. F. Suyagh, G. Iheagwaram, P. L. Kole et al., "Development and validation of a dried blood spot-HPLC assay for the determination of metronidazole in neonatal whole blood samples," Analytical and Bioanalytical Chemistry, vol. 397, no. 2, pp. 687693, 2010.

[44] R. Guthrie and A. Susi, "A simple phenylalanine method for detecting phenylketonuria in large populations of newborn infants," Pediatrics, vol. 32, pp. 338-343, 1963.

[45] I. Taneja, M. Erukala, K. S. R. Raju, S. P. Singh, and Wahajuddin, "Dried blood spots in bioanalysis of antimalarials: Relevance and challenges in quantitative assessment of antimalarial drugs," Bioanalysis, vol. 5, no. 17, pp. 2171-2186, 2013.

[46] P. Timmerman, S. White, S. Globig, S. Lüdtke, L. Brunet, and J. Smeraglia, "EBF recommendation on the validation of bioanalytical methods for dried blood spots," Bioanalysis, vol. 3, no. 14, pp. 1567-1575, 2011. 
[47] T. K. Majumdar and D. R. Howard, "The use of dried blood spots for concentration assessment in pharmacokinetic evaluations," Pharmacokinetics in Drug Development, vol. 3, pp. 91-114, 2011.

[48] G. Lawson, E. Cocks, and S. Tanna, "Bisoprolol, ramipril and simvastatin determination in dried blood spot samples using LC-HRMS for assessing medication adherence," Journal of Pharmaceutical and Biomedical Analysis, vol. 81-82, pp. 99-107, 2013.

[49] S. Tanna, D. Bernieh, and G. Lawson, "LC-HRMS analysis of dried blood spot samples for assessing adherence to cardiovascular medications," Journal of Bioanalysis \& Biomedicine, vol. 07, no. 01, 2015.

[50] D. Bernieh, G. Lawson, and S. Tanna, "Quantitative LC-HRMS determination of selected cardiovascular drugs, in dried blood spots, as an indicator of adherence to medication," Journal of Pharmaceutical and Biomedical Analysis, vol. 142, pp. 232-243, 2017.

[51] S. Tanna and G. Lawson, "Cardiovascular drug medication adherence assessed by dried blood spot analysis," Journal of Analytical \& Bioanalytical Techniques, vol. S12, article 006, 2014.

[52] K. Cvan Trobec, J. Trontelj, J. Springer, M. Lainscak, and M. Kerec Kos, "Liquid chromatography-tandem mass spectrometry method for simultaneous quantification of bisoprolol, ramiprilat, propranolol and midazolam in rat dried blood spots," Journal of Chromatography B, vol. 958, pp. 29-35, 2014.

[53] G. Lawson, H. Mulla, and S. Tanna, "Captopril determination in dried blood spot samples with LC-MS and LC-HRMS: A potential method for neonate pharmacokinetic studies," Journal of Bioanalysis \& Biomedicine, vol. 4, no. 2, pp. 16-25, 2012.

[54] R. N. Rao, S. Bompelli, and P. K. Maurya, "High-performance liquid chromatographic determination of anti- hypertensive drugs on dried blood spots using a fluorescence detector method development and validation," Biomedical Chromatography, vol. 25, no. 11, pp. 1252-1259, 2011.

[55] R. N. Rao, S. S. Raju, R. M. Vali, and G. G. Sankar, "Liquid chromatography-mass spectrometric determination of losartan and its active metabolite on dried blood spots," Journal of Chromatography B, vol. 902, pp. 47-54, 2012.

[56] J. M. Wiseman, C. A. Evans, C. L. Bowen, and J. H. Kennedy, "Direct analysis of dried blood spots utilizing desorption electrospray ionization (DESI) mass spectrometry," Analyst, vol. 135, no. 4, pp. 720-725, 2010.

[57] J. A. Ooms, L. Knegt, and E. H. M. Koster, "Exploration of a new concept for automated dried blood spot analysis using flowthrough desorption and online SPE-MS/MS," Bioanalysis, vol. 3, no. 20, pp. 2311-2320, 2011.

[58] J. Déglon, A. Thomas, A. Cataldo, P. Mangin, and C. Staub, “Online desorption of dried blood spot: A novel approach for the direct LC/MS analysis of $\mu$-whole blood samples," Journal of Pharmaceutical and Biomedical Analysis, vol. 49, no. 4, pp. 10341039, 2009.

[59] Z. Zhang, W. Xu, N. E. Manicke, R. G. Cooks, and Z. Ouyang, "Silica coated paper substrate for paper-spray analysis of therapeutic drugs in dried blood spots," Analytical Chemistry, vol. 84, no. 2, pp. 931-938, 2012.

[60] C. L. Bowen, M. D. Hemberger, J. R. Kehler, and C. A. Evans, "Utility of dried blood spot sampling and storage for increased stability of photosensitive compounds," Bioanalysis, vol. 2, no. 11, pp. 1823-1828, 2010.

[61] N. Kostić, Y. Dotsikas, N. Jović, G. Stevanović, A. Malenović, and M. Medenica, "Quantitation of pregabalin in dried blood spots and dried plasma spots by validated LC-MS/MS methods," Journal of Pharmaceutical and Biomedical Analysis, vol. 109, pp. 79-84, 2015.

[62] Y. Li, J. Henion, R. Abbott, and P. Wang, "Dried blood spots as a sampling technique for the quantitative determination of guanfacine in clinical studies," Bioanalysis, vol. 3, no. 22, pp. 2501-2514, 2011.

[63] Y. Li, J. Henion, R. Abbott, and P. Wang, "Semi-automated direct elution of dried blood spots for the quantitative determination of guanfacine in human blood," Bioanalysis, vol. 4, no. 12, pp. 1445-1456, 2012.

[64] Y. Li, J. Henion, R. Abbott, and P. Wang, "The use of a membrane filtration device to form dried plasma spots for the quantitative determination of guanfacine in whole blood," Rapid Communications in Mass Spectrometry, vol. 26, no. 10, pp. 1208-1212, 2012.

[65] M. L. Della Bona, S. Malvagia, F. Villanelli et al., "A rapid liquid chromatography tandem mass spectrometry-based method for measuring propranolol on dried blood spots," Journal of Pharmaceutical and Biomedical Analysis, vol. 78-79, pp. 34-38, 2013.

[66] A. Thomas, H. Geyer, W. Schänzer et al., "Sensitive determination of prohibited drugs in dried blood spots (DBS) for doping controls by means of a benchtop quadrupole/Orbitrap mass spectrometer," Analytical and Bioanalytical Chemistry, vol. 403, no. 5, pp. 1279-1289, 2012.

[67] G. T. Clark, G. Giddens, L. Burrows, and C. Strand, "Utilization of dried blood spots within drug discovery: Modification of a standard DiLab ${ }^{\circledR}$ AccuSampler ${ }^{\circledR}$ to facilitate automatic dried blood spot sampling," Laboratory Animals, vol. 45, no. 2, pp. 124-126, 2011.

[68] H. Huang, Q. Wu, L. Zeng et al., "Heating paper spray mass spectrometry for enhanced detection of propranolol in dried blood samples," Analytical Methods, vol. 9, no. 29, pp. 42824287, 2017.

[69] X. Liang, H. Jiang, and X. Chen, "Human DBS sampling with LC-MS/MS for enantioselective determination of metoprolol and its metabolite O-desmethyl metoprolol," Bioanalysis, vol. 2, no. 8, pp. 1437-1448, 2010.

[70] N. Ganz, M. Singrasa, L. Nicolas et al., "Development and validation of a fully automated online human dried blood spot analysis of bosentan and its metabolites using the Sample Card And Prep DBS System," Journal of Chromatography B, vol. 885886, pp. 50-60, 2012.

[71] M. Géhin, P. N. Sidharta, and J. Dingemanse, "Bosentan Pharmacokinetics in Pediatric Patients with Pulmonary Arterial Hypertension: Comparison of Dried Blood Spot and Plasma Analysis," Pharmacology, vol. 98, no. 3-4, pp. 111-114, 2016.

[72] Y. Enderle, A. D. Meid, J. Friedrich et al., "Dried Blood Spot Technique for the Monitoring of Ambrisentan, Bosentan, Sildenafil, and Tadalafil in Patients with Pulmonary Arterial Hypertension," Analytical Chemistry, vol. 87, no. 24, pp. 1211212120, 2015.

[73] P. D. Rainville, J. L. Simeone, D. S. Root, C. R. Mallet, I. D. Wilson, and R. S. Plumb, "A method for the direct injection and analysis of small volume human blood spots and plasma extracts containing high concentrations of organic solvents using revered-phase 2D UPLC/MS," Analyst, vol. 140, no. 6, pp. 1921-1931, 2015.

[74] M. V. Antunes, M. F. Charão, and R. Linden, "Dried blood spots analysis with mass spectrometry: Potentials and pitfalls 
in therapeutic drug monitoring," Clinical Biochemistry, vol. 49, no. 13-14, pp. 1035-1046, 2016.

[75] J. Déglon, L. A. Leuthold, and A. Thomas, "Potential missing steps for a wide use of dried matrix spots in biomedical analysis," Bioanalysis, vol. 7, no. 18, pp. 2375-2382, 2015.

[76] C. Borghi, A. Dormi, M. Veronesi, V. Immordino, and E. Ambrosioni, "Use of Lipid-Lowering Drugs and Blood Pressure Control in Patients With Arterial Hypertension," The Journal of Clinical Hypertension, vol. 4, no. 4, pp. 277-285, 2002.

[77] J. E. Rodgers and J. H. Patterson, "The role of the reninangiotensin-aldosterone system in the management of heart failure," Pharmacotherapy, vol. 20, no. 11, part 2, pp. 368S-378S, 2000.

[78] J. H. Bauer, "The angiotensin II type 1 receptor antagonists. A new class of antihypertensive drugs," JAMA Internal Medicine, vol. 155, no. 13, pp. 1361-1368, 1995.

[79] J. Nie, M. Zhang, Y. Fan, Y. Wen, B. Xiang, and Y.-Q. Feng, "Biocompatible in-tube solid-phase microextraction coupled to HPLC for the determination of angiotensin II receptor antagonists in human plasma and urine," Journal of Chromatography $B$, vol. 828, no. 1-2, pp. 62-69, 2005.

[80] M. Polinko, K. Riffel, H. Song, and M.-W. Lo, "Simultaneous determination of losartan and EXP3174 in human plasma and urine utilizing liquid chromatography/tandem mass spectrometry," Journal of Pharmaceutical and Biomedical Analysis, vol. 33, no. 1, pp. 73-84, 2003.

[81] N. D. S. Kostić, Y. Dotsikas, A. D. S. Malenović, and M. Medenica, "Effects of derivatization reagents consisting of nalkyl chloroformate/n-alcohol combinations in LC-ESI-MS/MS analysis of zwitterionic antiepileptic drugs," Talanta, vol. 116, pp. 91-99, 2013.

[82] R. J. W. Meesters and G. P. Hooff, "State-of-the-art dried blood spot analysis: An overview of recent advances and future trends," Bioanalysis, vol. 5, no. 17, pp. 2187-2208, 2013.

[83] Y. Enderle, K. Foerster, and J. Burhenne, "Clinical feasibility of dried blood spots: Analytics, validation, and applications," Journal of Pharmaceutical and Biomedical Analysis, vol. 130, pp. 231-243, 2016.

[84] M. Wagner, D. Tonoli, E. Varesio, and G. Hopfgartner, "The use of mass spectrometry to analyze dried blood spots," Mass Spectrometry Reviews, vol. 35, no. 3, pp. 361-368, 2016.

[85] P. Denniff and N. Spooner, "The effect of hematocrit on assay bias when using DBS samples for the quantitative bioanalysis of drugs," Bioanalysis, vol. 2, no. 8, pp. 1385-1395, 2010.

[86] R. Sturm, J. Henion, R. Abbott, and P. Wang, "Novel membrane devices and their potential utility in blood sample collection prior to analysis of dried plasma spots," Bioanalysis, vol. 7, no. 16, pp. 1987-2002, 2015.

[87] B. G. Keevil, "The analysis of dried blood spot samples using liquid chromatography tandem mass spectrometry," Clinical Biochemistry, vol. 44, no. 1, pp. 110-118, 2011.

[88] T. Panchal, N. Spooner, and M. Barfield, "Ensuring the collection of high-quality dried blood spot samples across multisite clinical studies," Bioanalysis, vol. 9, no. 2, pp. 209-213, 2017. 

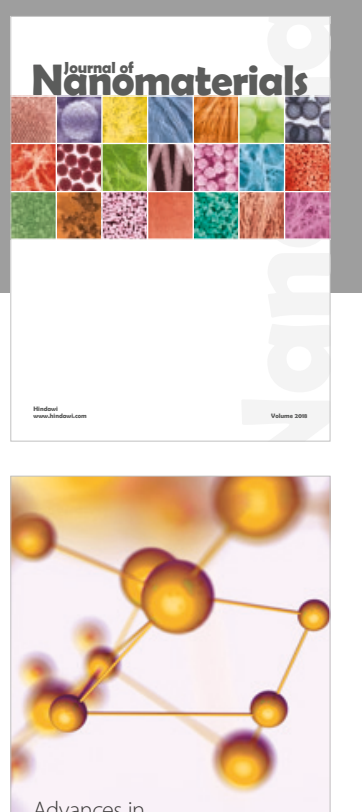

Physical Chemistry
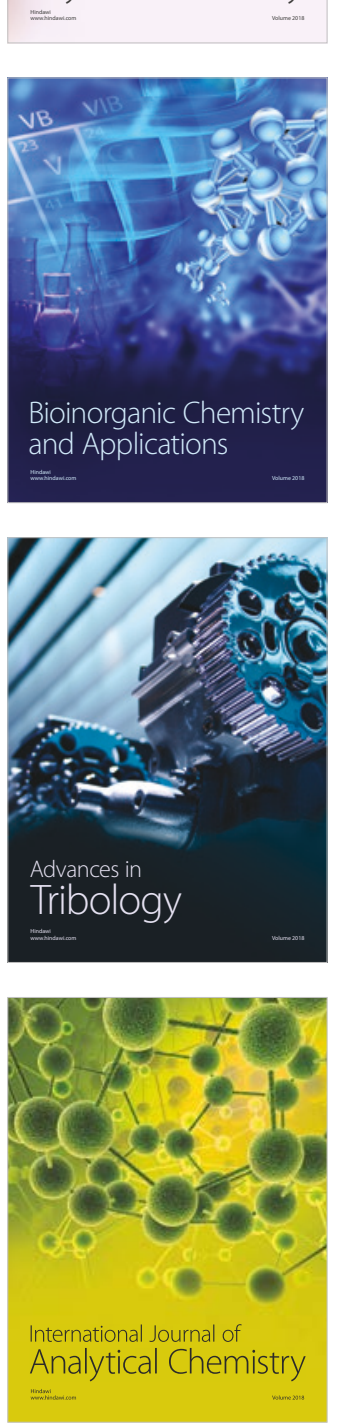

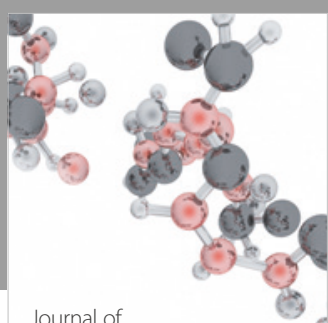

Analytical Methods

in Chemistry

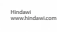

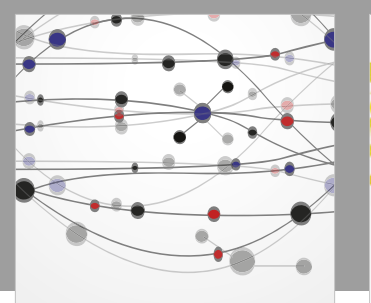

The Scientific World Journal

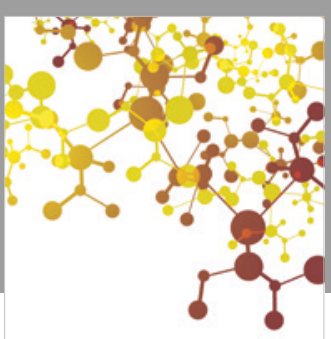

Journal of

Applied Chemistry
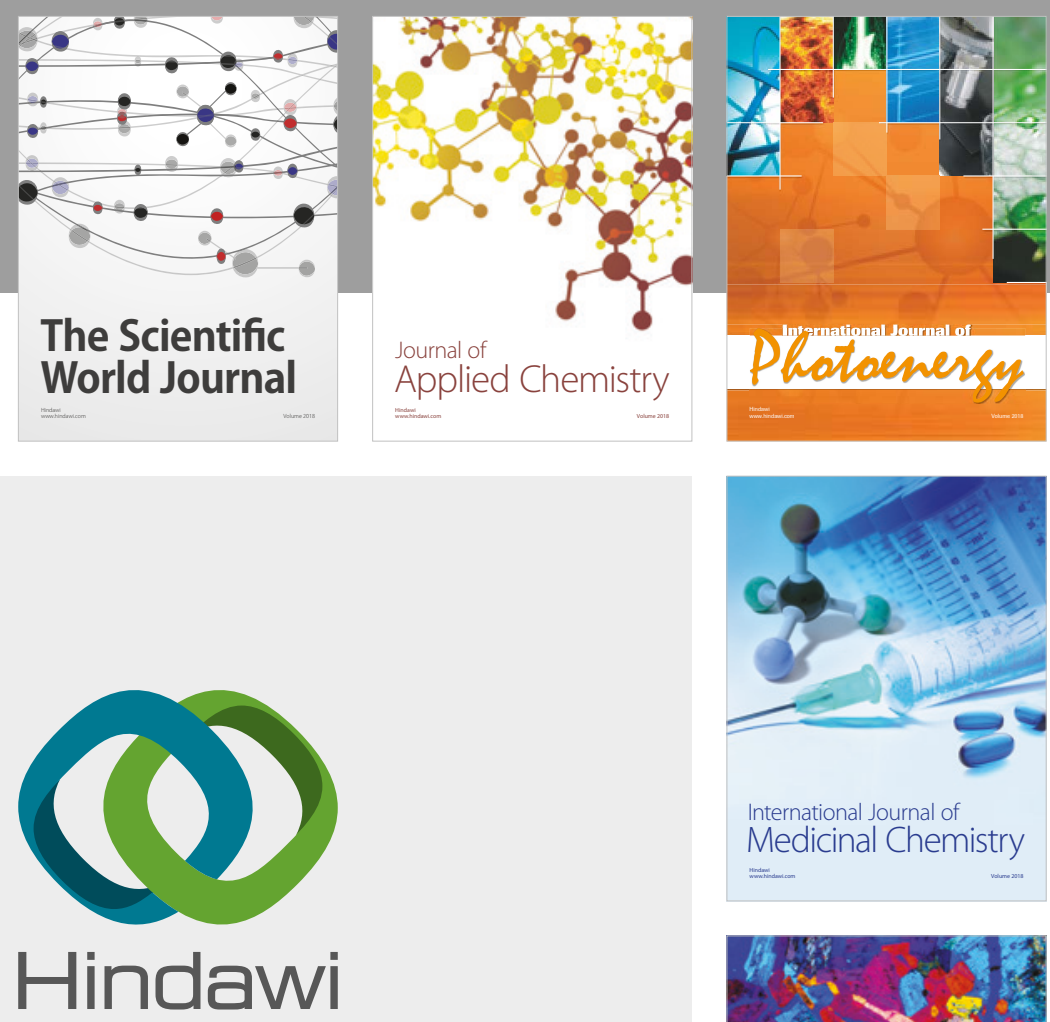

Submit your manuscripts at

www.hindawi.com
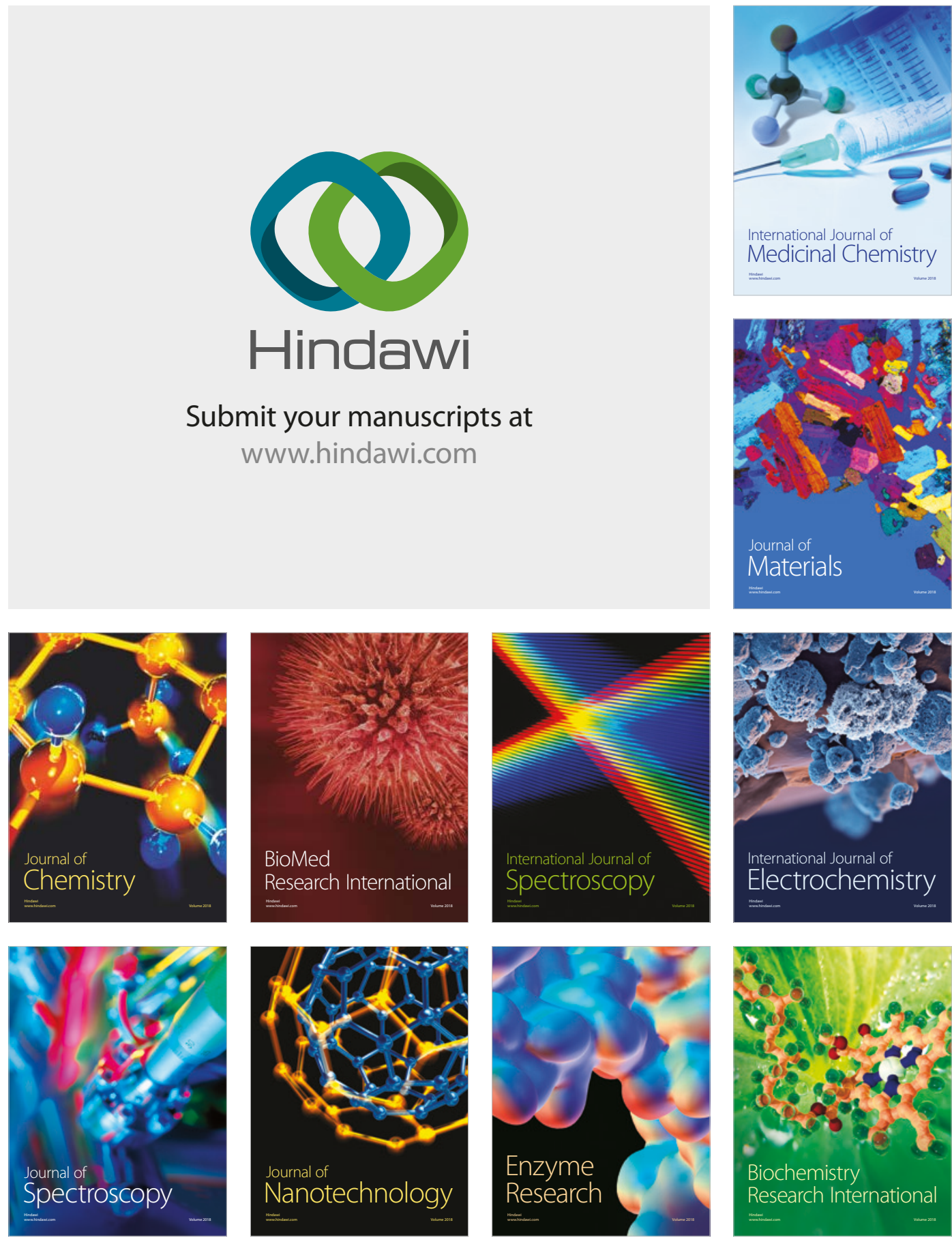
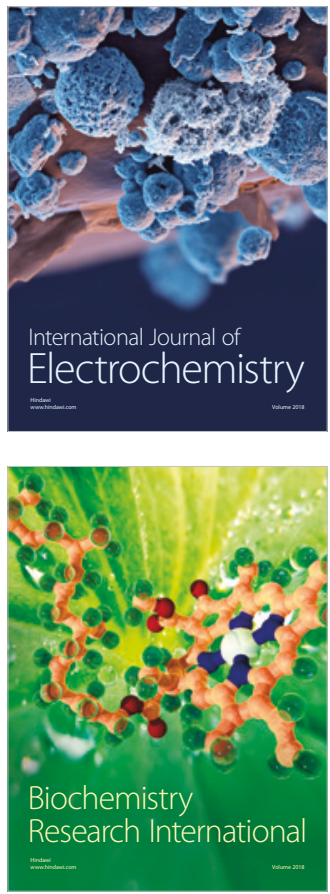